\title{
miR-126 suppresses epithelial-to-mesenchymal transition and metastasis by targeting PI3K/AKT/Snail signaling of lung cancer cells
}

\author{
ZHIJUN JIA $^{1}$, YING ZHANG ${ }^{2}$, QINFENG XU ${ }^{1}$, WANHUA GUO ${ }^{1}$ and AIBIN GUO ${ }^{3}$ \\ ${ }^{1}$ Department of Nuclear Medicine, ${ }^{2}$ Prenatal Diagnosis Center and ${ }^{3}$ Department of Geriatrics, The Affiliated \\ Drum Tower Hospital of Nanjing University Medical School, Nanjing, Jiangsu 210008, P.R. China
}

Received June 10, 2016; Accepted February 7, 2018

DOI: $10.3892 / \mathrm{ol} .2018 .8207$

\begin{abstract}
Although previous studies have demonstrated that dysregulation of microRNA (miR)-126 is associated with the progression of several types of cancer, including lung cancer, the relationship between miR-126 and lung cancer metastasis remains unclear. SPC-A1 lung cancer cells were transfected with miR-126 mimic and negative control using Lipofectamine $^{\circledR} 3000$. Following 2 h, TGF- $\beta 1$ was used to induce epithelial-to-mesenchymal transition (EMT). The protein expression levels of EMT markers: E-cadherin, fibronectin, $\mathrm{N}$-cadherin and vimentin were detected by western blot analysis or immunofluorescence staining. The results demonstrated that ectopic expression of miR-126 significantly suppresses the epithelial-to-mesenchymal transition process, which is considered to be the initial step of tumor metastasis, in SPC-A1 lung cancer cells. In addition, lentivirus-delivered miR-126 was demonstrated to endow Lewis lung carcinoma (LLC) cells with the ability to suppress lung metastasis in vivo. Previous studies have demonstrated that the molecular signals for this phenomenon involve the inhibition of the phosphoinositide 3-kinase/protein kinase B/Snail pathway by miR-126. The protein levels of p-PDK1 (S241) and p-AKT (S473) decreased in miR-126 mimic transfected SPC-A1 and LLC cells, compared with the control group, which were detected by western blot analysis. Reverse transcription-quantitative polymerase chain reaction and western blot analysis results indicated that the expression of Snail decreased in miR-126 mimic transfected SPC-A1 and LLC cells. In conclusion, these results revealed an important role for miR-126 in the regulation of the invasive and metastatic potential of lung
\end{abstract}

Correspondence to: Dr Wanhua Guo, Department of Nuclear Medicine, The Affiliated Drum Tower Hospital of Nanjing University Medical School, 321 Zhongshan Road, Nanjing, Jiangsu 210008, P.R. China

E-mail: wanhuaguo@163.com

Key words: microRNA-126, epithelial-to-mesenchymal transition, metastasis, lung cancer cancer, and suggested a potential application for miR-126 in lung cancer treatment.

\section{Introduction}

Lung cancer is the most common cause of cancer-associated mortality globally (1). Despite improvements in early diagnosis and novel therapies, the 5-year survival rate for patients with lung cancer is still $<15 \%$. The major cause of the low survival rate of patients with lung cancer is distant metastasis (2). Previous studies have demonstrated that epithelial-to-mesenchymal transition (EMT), which is characterized by the loss of epithelial markers and the gain of a mesenchyme-like phenotype, serves a key function in the early process of cancer cell metastasis $(3,4)$. During the transition, cancer cells lose the expression of genes that promote cell-cell contact, including epithelial cadherin (E-cadherin), and gain the expression of mesenchymal markers, including vimentin, fibronectin and neural cadherin (N-cadherin). Transited tumor cells are thought to have increased potential for motility, invasion and metastasis, as well as increased resistance to chemotherapy (5-7). A group of transcription factors has been confirmed to be capable of orchestrating EMT programs in cancer progression. These include direct transcriptional repressors of E-cadherin, including Snail, Slug, zinc finger E-box binding homeobox (ZEB)1, ZEB2 and others, including Twist-related protein 1 (Twist1) and forkhead box C2 (8).

MicroRNAs (miRNAs/miRs) represent a group of small non-coding RNAs (18-25 nucleotides) that induce translational repression or target degradation by binding to the 3'-untranslated regions of target mRNAs (9). miRNAs are involved in almost every biological process, including the cell cycle, growth, apoptosis, differentiation and stress responses, and the regulation of gene expression (10). Furthermore, evidence indicates that certain miRNAs may function as tumor suppressors or oncogenes, potentially contributing to tumorigenesis by binding to their targets $(9,11,12)$. miR-126 has frequently been reported to be downregulated in human cancers, including osteosarcoma, breast cancer, pancreatic cancer and colorectal cancer (13-15). In these types of cancer, miR-126 inhibits cancer cell growth by binding to targets including insulin receptor substrate 1 , phosphoinositide 3 -kinase regulatory 
subunit 2, ADAM metallopeptidase domain 9, vascular endothelial growth factor and certain other molecules (16-18). Furthermore, certain studies have demonstrated that miR-126 acts as a negative regulator of tumor invasion and metastasis in breast cancer $(18,19)$. In lung cancer cells, miR-126 also inhibits cell proliferation and invasion (20-23), but the function of miR-126 in lung cancer metastasis, its role in patients with lung cancer and in murine lung cancer models, as well as the mechanisms by which miR-126 performs its functions and modulates the malignant phenotypes of lung cancer cells, remain to be fully understood.

The present study demonstrated that overexpression of miR-126 inhibited transforming growth factor- $\beta 1$ (TGF- $\beta 1$ )-induced EMT. Furthermore, stable ectopic expression of miR-126 inhibited the metastasis of lung cancer cells in a mouse xenograft model, suggesting that EMT inhibition may be a central mechanism that suppresses cancer invasion and metastasis. Furthermore, the present study identified that the inhibitory function of miR-126 may be achieved by targeting the phosphoinositide 3-kinase (PI3K)/protein kinase $\mathrm{B}(\mathrm{AKT}) /$ Snail signaling pathway. Thus, the results of the present study provide valuable insights toward developing more effective clinical therapies for lung cancer in the future.

\section{Materials and methods}

Cell culture and transfections. The SPC-A1 and LLC cell lines were purchased from the Cell Bank of Type Culture Collection of the Chinese Academy of Sciences (Shanghai, China). The cells were cultured in RPMI-1640 medium (Gibco; Thermo Fisher Scientific, Inc., Waltham, MA, USA), supplemented with $10 \%$ fetal bovine serum (FBS; Hyclone; GE Healthcare Life Sciences, Logan, UT, USA), and maintained at $37^{\circ} \mathrm{C}$ in a humidified environment containing $5 \% \mathrm{CO}_{2}$. SPC-A1 cells were seeded into 6 -well plates with $2.5 \times 10^{4}$ cells/well. Cells were grown to $70 \%$ confluence, serum-starved for $24 \mathrm{~h}$, and then treated with human recombinant TGF- $\beta 1$ ( $5 \mathrm{ng} / \mathrm{ml}$; cat. no. 100-21; PeproTech. RockyHill, NJ, USA) or equal amounts of dimethyl sulfoxide (DMSO) for $48 \mathrm{~h}$ at $37^{\circ} \mathrm{C}$. For LY294002 treatment, $24 \mathrm{~h}$ after seeding, the medium was removed and replaced with LY294002 (50 $\mu \mathrm{mol} / 1$; cat. no. L9908; Sigma-Aldrich; Merck KGaA, Darmstadt, Germany) or equal amounts of DMSO and incubated for $48 \mathrm{~h}$ at $37^{\circ} \mathrm{C}$. miR-126 mimics (5'-UCGUACCGUGAGUAAUAAUGCG-3') and its scramble control (5'-UAGUCAACGAGUCUAUGAGUC G-3') were designed and chemically synthesized by RiboBio Co., Ltd (Guangzhou, China). For transfection, the cells were plated into 6 -well plates with $2.5 \times 10^{4}$ cells/well. Once the cells were $30-50 \%$ confluent, miR-126 mimics or NC mimics were transfected into the SPC-A1 cells using Lipofectamine ${ }^{\circledR} 3000$ reagent (Invitrogen; Thermo Fisher Scientific, Inc.), according to the manufacturer's protocol.

Reverse transcription-quantitative polymerase chain reaction (RT-qPCR) analysis. Total RNA was extracted from SPC-A1 cells with TRIzol ${ }^{\circledR}$ (Invitrogen; Thermo Fisher Scientific Inc.). A total of $1 \mu \mathrm{g}$ RNA was transcribed into cDNA using 1st Strand cDNA Synthesis Kit (Vazyme Biotech Co., Ltd., Nanjing, China) for mRNA and an RT-qPCR kit (Takara Bio Inc., Otsu, Japan) for miRNA according to the manufacturer's protocol. The expression levels of the genes were detected by qPCR. The following primers were used: Human (h-) miR-126 forward, 5'-GTCGTATCCAGTGCA GGGTCCGAG-3' and reverse, 5'-GTATTCGCACTGGAT ACGAC-3'; h-U6 forward, 5'-CTCGCTTCGGCAGCA CA-3' and reverse, 5'-AACGCTTCACGAATTTGCGT-3'; h-Snail forward, 5'-TCGGAAGCCTAACTACAGCGA-3' and reverse, 5'-AGATGAGCATTGGCAGCGAG-3'; h-Twist1 forward, 5'-TGCAGACGCAGCGGGTCATG-3' and reverse, 5'-GGACCGGCGGTCGAACTCCC-3'; h-ZEB1 forward, 5'-GATGATGAATGCGAGTCAGATGC-3' and reverse, 5'-ACAGCAGTGTCTTGTTGTTGT-3'; h-ZEB2 forward, 5'-CAAGAGGCGCAAACAAGCC-3' and reverse, 5'-GGT TGGCAATACCGTCATCC-3'; E-cadherin forward, 5'-CAG CCTGTCGAAGCAGGATTGC-3' and reverse, 5'-GAGCTC AGACTAGCAGCTTCGG-3'. qPCR was performed using the SYBR Green (Takara Biotechnology Co. Ltd, Dalian, China) dye detection method on the ABI PrismStep-One Plus instrument (Applied Biosystems; Thermo Fisher Scientific, Inc.). The thermocycling conditions were as follows: $95^{\circ} \mathrm{C}$ for $30 \mathrm{sec}$; followed by 40 cycles of $95^{\circ} \mathrm{C}$ for $5 \mathrm{sec}$; and $60^{\circ} \mathrm{C}$ for $30 \mathrm{sec}$. The comparative $\mathrm{Cq}$ method was used for quantification of the transcripts (24).

Immunofluorescent (IF) staining. For IF staining, the SPC-A1 cells grown on the slides were fixed with $4 \%$ paraformaldehyde for $30 \mathrm{~min}$ at $4^{\circ} \mathrm{C}$, then blocked with $5 \%$ bovine serum album in for $1 \mathrm{~h}$ at room temperature and incubated with primary antibodies overnight at $4^{\circ} \mathrm{C}$. The next day, the slides were washed with PBS, and incubated with Cy3-goat anti-rabbit secondary antibodies (1:200; cat. no. 111-165-003; Jackson Immuno Research Laboratories, West Grove, PA, USA) and DAPI (1 mg/ml; cat. no. D6584; Sangon Biotech Co., Ltd., Shanghai, China) for $2 \mathrm{~h}$ at room temperature. The following primary antibodies were used: E-cadherin (1:200; cat. no. ab1416; Abcam, Cambridge, UK), Fibronectin (1:200; cat. no. ab2413; Abcam), Ki-67 (1:100; cat. no. GA626; Dako; Agilent Technologies, Inc., Santa Clara, CA, USA). Fluorescence microscopy images were obtained with a research fluorescence microscope (Olympus Corporation, Tokyo, Japan) equipped with a digital camera (at least 5 fields of view at x400 magnification).

Western blotting. Cells or tumors were lysed in RIPA buffer supplemented with complete Protease Inhibitor Cocktail tablets (Roche, Inc., Basel, Switzerland) for $30 \mathrm{~min}$ on ice. Protein lysates $(30 \mu \mathrm{g})$ were subjected to $10 \%$ SDS-PAGE (Pierce; Thermo Fisher Scientific, Inc.) and transferred to PVDF membrane. Subsequent to blocking with 5\% non-fat milk in $0.05 \%$ TBS-Tween-20 (v/v) for $1 \mathrm{~h}$ at room temperature, the membranes were incubated with the appropriate primary antibodies overnight at $4^{\circ} \mathrm{C}$. Primary antibodies used were as follows: Anti-E-cadherin (1:1,000; cat. no. 3195; Cell Signaling Technology, Inc.), N-cadherin (1:2,000; cat. no. 4061; Cell Signaling Technology, Inc.), vimentin (1:1,000; cat. no. 3932; Cell Signaling Technology, Inc.), phosphorylated (p-)AKT (ser-473; 1:2,000; cat. no. 2118-1; Epitomics; Abcam), p-PDK1 (ser-241; 1:500; cat. no. ab131067; Abcam) and $\beta$-actin (1:5,000; cat. no. MS-1295-P; Pierce; Thermo Fisher Scientific, Inc.). The secondary antibodies were horseradish peroxidase 
(HRP)-conjugated goat anti-mouse IgG (cat. no. AP308P; heavy + light; 1:3,000; Sigma-Aldrich; Merck KGaA) and HRP-conjugated goat anti-rabbit IgG (cat. no. AP307P; heavy + light; 1:3,000; Sigma-Aldrich; Merck KGaA). The secondary antibodies were incubated for $1 \mathrm{~h}$ at room temperature. Protein detection was performed using an enhanced chemiluminescence substrate (Thermo Fisher Scientific, Inc.) prior to exposure to film.

Stable overexpression of miR-126 in lung cancer cells and mouse xenograft assay. The oligonucleotide sequence of miR-126 (5'-UCGUACCGUGAGUAAUAAUGCG-3') and scramble control (5'-UAGUCAACGAGUCUAUGAGUC G-3') were synthesized and cloned intopGFP-C-shLentia lentiviral vector (cat. no. TR30023; OriGene Technologies, Inc., Rockville; MD, USA) as previously reported (25). The following primers were used: miR-126 forward, 5'-TAT CTTGTGGAAAGGACGCG-3' and reverse, 5'-AGACGT TCCAAAAAATCGTACCGTGAGTAATAATGCGTCAAG AGCGCATTATTACTCACGGTACGAGTCTTCTGACGC TGCTGCCTG-3'; scramble control forward, 5'-TATCTTGTG GAAAGGACGCG-3' and reverse, 5'-AGACGTTCCAAA AAATAGTCAACGAGTCTATGAGTCGTCAAGAGCGAC TCATAGACTCGTAGTCTAGTCTTCTGACGCTGCTGC CTG-3'; Advantage GC 2 polymerase mix (cat. no. 639114; Clontech Laboratories, Inc., Mountainview, CA, USA) was used for amplification. For lentiviral infection, non-transfected LLC cells were eliminated by 2 week spuromycin incubation at $37^{\circ} \mathrm{C}$. The overexpression of miR-126 in puromycin-resistant LLC cells was verified by RT-qPCR as previously mentioned.

Female C57BL/6 mice were $(n=8)$ purchased from the Model Animal Research Center of Nanjing University (Nanjing, China; age, 4-6 weeks; weight, 15-18 g). Mice were bred in an SPF room of the core animal facility of the Model Animal Research Center of Nanjing University (Nanjing, China). The room temperature was $25^{\circ} \mathrm{C}$ and the light-cycle is automatically controlled ( $12 \mathrm{~h}$ light and $12 \mathrm{~h}$ dark). Mice had free access to food and water. LLC cells $\left(1 \times 10^{6}\right)$ stably overexpressing miR-126 or NC were suspended with $100 \mu \mathrm{l}$ $50 \%$ Matrigel and injected subcutaneously into one side of the flanks of the mice. Mice were anesthetized by intraperitoneal injection with fresh 2,2,2-tribromoethanol (Avertin; cat. no. B65586; Sigma-Aldrich; Merck KGaA) in sterile PBS at a dose of $0.4 \mathrm{mg} / \mathrm{g}$. Primary tumors were surgically removed 1 week following transplantation or when reaching a volume of $800 \mathrm{~mm}^{3}$ to allow further growth of metastatic nodules. Following 3 weeks of tumor growth, the mice were sacrificed and metastasis to the lung was determined by counting individual metastatic nodules. The results are presented as the mean \pm standard error of the mean (SEM).

Hematoxylin-eosin $(H \& E)$ staining. Mouse lungs were dissected and washed with cold PBS and fixed in $4 \%$ paraformaldehyde overnight at $4^{\circ} \mathrm{C}$. The samples were processed successively by: i) a 30 min wash in PBS at $4^{\circ} \mathrm{C}$; ii) an $1 \mathrm{~h}$ incubation in 70, 80 and $95 \%$ ethanol and a $1 \mathrm{~h}$ incubation in $100 \%$ ethanol at room temperature; iii) a 20 min incubation in xylene at room temperature; iv) a $1 \mathrm{~h}$ incubation in paraffin/xylene $(1: 1)$ at $65^{\circ} \mathrm{C}$; and v) a $1 \mathrm{~h}$ incubation in fresh paraffin at $65^{\circ} \mathrm{C}$. The processed samples were then embedded in paraffin and sectioned ( $8 \mu \mathrm{m}$ thick). The sections were applied for H\&E staining: Briefly, sections were washed in distilled water and incubated with $0.2 \%$ hematoxylin solution (w/v) for $5 \mathrm{~min}$. Differentiation followed for $30 \mathrm{sec}$ in $1 \%$ acid alcohol $(1 \% \mathrm{HCl}$ in $70 \%$ Ethanol). Subsequently, the sections were stained with $0.25 \%$ eosin (w/v) for $15 \mathrm{sec}$ and dehydrated with an alcohol series (70, 80, 90 and 100\%) for $2 \mathrm{~min}$ each. The sections were visualized under a light microscope (magnification, x6).

Statistical analysis. Each value in the present study was obtained from at least three independent experiments. Data were presented as the mean \pm SEM. Since two groups were being compared in the present study, statistical significance was determined using the unpaired two-tailed Student's t-test. Statistical analysis was performed using GraphPad Prism 5.0 software (GraphPad Software, Inc., La Jolla, CA, USA). $\mathrm{P}<0.05$ was considered to indicate a statistically significant difference.

\section{Results}

miR-126 inhibits the EMT of lung cancer cells in vitro. EMT is an important process involved in the invasion and metastasis of tumor cells. To assess whether miR-126 affects the EMT of lung cancer cells, miR-126 mimics were used to overexpress miR-126 in the human lung cancer SPC-A1 cell line. The expression of miR-126 was verified by RT-qPCR (Fig. 1A). Cells were treated with TGF- $\beta 1$ $(5 \mathrm{ng} / \mathrm{ml}) 2 \mathrm{~h}$ following transfection with miR-126 mimics and NC. TGF- $\beta 1$ is widely used to induce EMT in epithelial cells (26). Immunofluorescent staining of fibronectin and E-cadherin revealed that TGF- $\beta 1$-treated control cells exhibited loss of tight junctions and epithelial phenotypes while acquiring spindle-like, fibroblastic morphology (Fig. 1B). However, miR-126 overexpression inhibited EMT-associated changes (Fig. 1B). The relative protein expression of EMT markers was also measured by western blot analysis. Upregulation of miR-126 expression in SPC-A1 cells resulted in a visible increase in E-cadherin expression and a decrease in $\mathrm{N}$-cadherin and vimentin expression compared with control cells (Fig. 1C). These results indicated that ectopic expression of miR-126 inhibited the EMT phenotype in SPC-A1 cells.

miR-126 inhibits metastasis of lung cancer cells in vivo. To directly evaluate the function of miR-126 in tumor metastasis in vivo, a xenograft LLC model in C57BL/6 mice was used. LLC cells transfected with miR-126 or scramble control lentivirus were injected subcutaneously into each flank of the mice. Primary tumors were surgically removed $\sim 1$ week following injection. There was no significant difference in the size and weight of primary tumors between these two groups (Fig. 2A and B).Immunofluorescent staining of the Ki-67 proliferation marker also confirmed these results (Fig. 2C and D). To examine whether miR-126 inhibited the EMT of lung cancer cells in vivo, the expression of EMT markers was examined by western blot analysis. Consistent with the in vitro results, increased expression of E-cadherin and decreased expression of $\mathrm{N}$-cadherin and vimentin were observed in the miR-126 
A

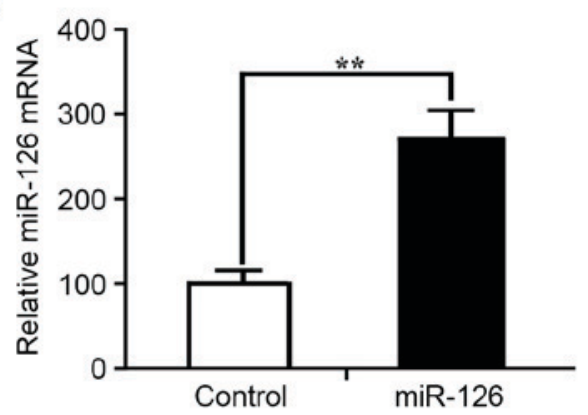

C
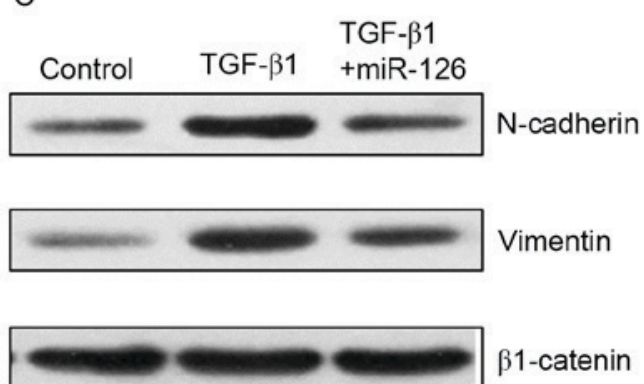

B
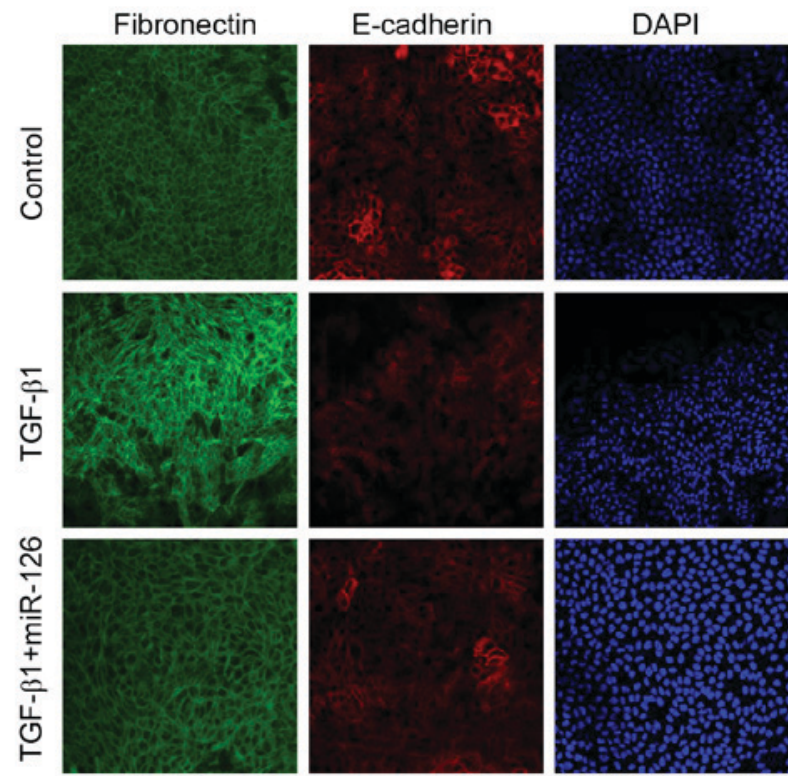

Figure 1. miR-126 inhibits the EMT of lung cancer cells in vitro. (A) SPC-A1 lung cancer cells were transfected with miR-126 mimics or negative control mimics for $48 \mathrm{~h}$. Overexpression of miR-126 was validated with reverse transcription-quantitative polymerase chain reaction. (B) Immunofluorescent staining of fibronectin (green) and E-cadherin (red) in SPC-A1 cells (magnification, x400). Nucleiwere stained with DAPI (blue). SPC-A1 cells were treated with TGF- $\beta 1(5 \mathrm{ng} / \mathrm{ml})$ for $48 \mathrm{~h}$. Cells without any treatment were used as the normal control. (C) Western blot analysis of N-cadherin and vimentin expression in SPC-A1 cells. $\beta$-actin was used as an internal control. ${ }^{* *} \mathrm{P}<0.05$ vs. control. EMT, epithelial-mesenchymal-transition; miR, microRNA; E-cadherin, epithelial cadherin; N-cadherin, neural cadherin; TGF- $\beta 1$, transforming growth factor- $\beta 1$.

A

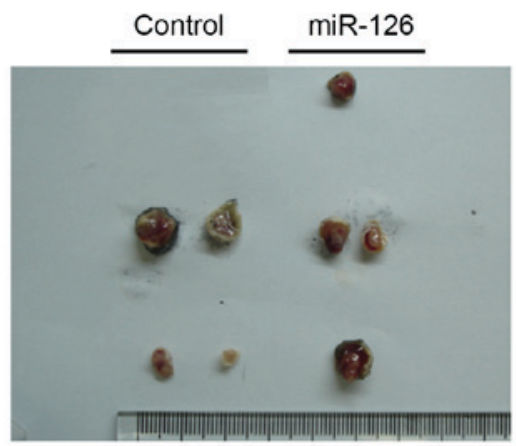

C

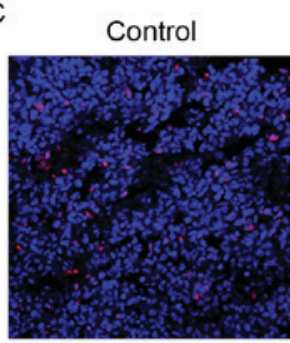

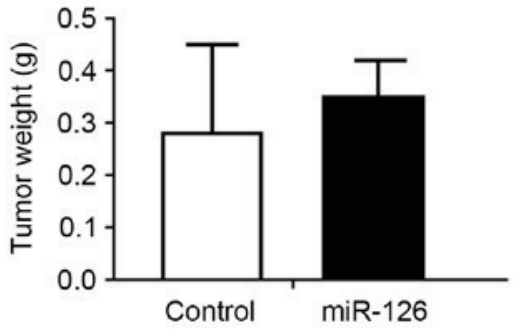

D

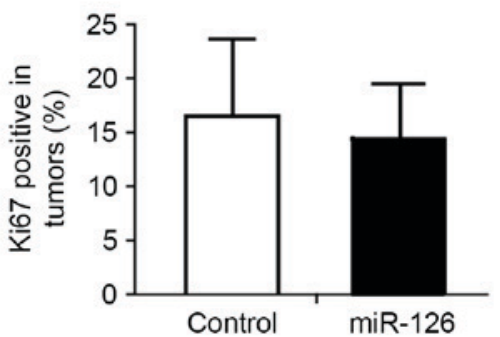

Figure 2. miR-126 has no effect on the proliferation of lung cancer cells in vivo. (A) LLC cell-derived primary tumors 1 week following subcutaneous injection (magnification, x1). (B) Average weight of the LLC-derived primary tumors obtained from control and miR-126 overexpression mice. (C) Ki-67 staining (red) with the primary tumor sections. Nuclei were stained with DAPI (blue) (magnification, x400). (D) Quantification of Ki-67 positive cells in the primary tumor sections. miR, microRNA.

overexpression group (Fig. 3A). Stable ectopic miR-126 expression significantly suppressed the formation of lung metastases 3 weeks following the surgical removal of the primary tumors
(Fig. 3B and C). These results indicated that miR-126 maybe capable of repressing lung cancer metastasis by preventing the EMT process in vivo. 
A

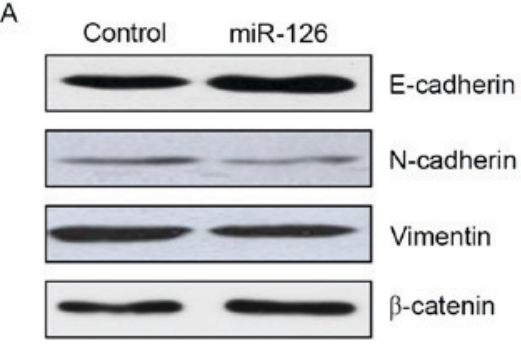

C

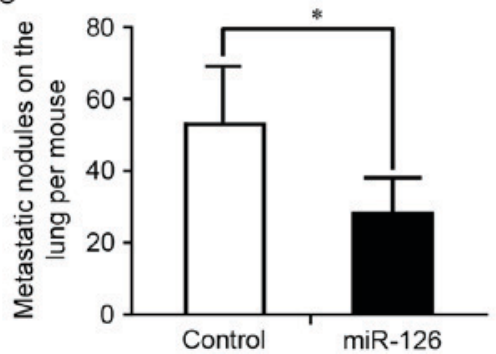

B
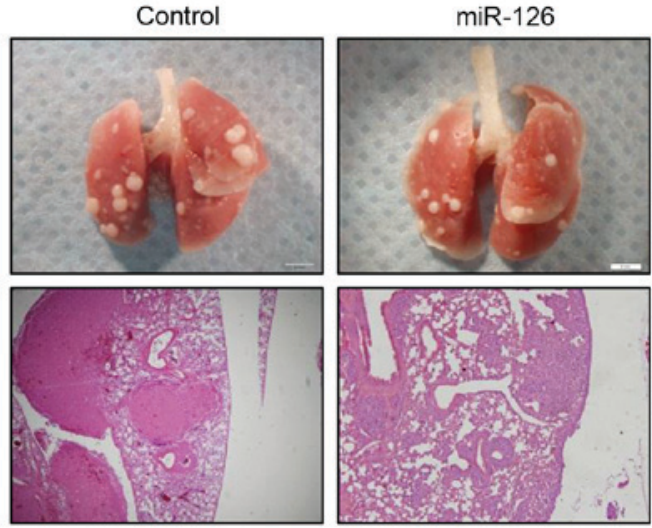

Figure 3. miR-126 inhibits metastasis of lung cancer cells in vivo. (A) Western blot analysis of E-cadherin, N-cadherin and vimentin expression in LLC-derived primary tumors. $\beta$-actin was used as an internal control. (B) Representative metastases (upper) and morphology of the lung (lower) 3 weeks following removal of the primary tumor. (C) Quantification of lung metastases of the control and miR-126-overexpression group. " $\mathrm{P}<0.05$ vs. control. miR, microRNA; E-cadherin, epithelial cadherin; N-cadherin, neural cadherin.

The PI3K/AKT/Snail pathway is a direct target of miR-126 in lung cancer cells. Previous studies have demonstrated that multiple predicted and validated miR-126 targets are signaling inputs for AKT activity $(27,28)$. To investigate whether the inhibitory function of miR-126 on the metastasis of lung cancer cells is mediated by the PI3K/AKT pathway, the activity of this pathway was examined following miR-126 overexpression. p-PDK1 and p-AKT were significantly downregulated in SPC-A1 and LLC cells overexpressing miR-126 (Fig. 4A and B). Expression of the transcription factor Snail was reduced in the two miR-126 transfected cell lines compared with the NC cells, but Slug, Twist1 and ZEB1 were not affected (Fig. 4C and D). Constitutively active AKT induces Snail expression (29). To examine whether reduced Snail expression accounted for the reduced PI3K/AKT activity, LY294002 was used to treat SPC-A1 and the LLC cells. Snail mRNA expression was reduced following treatment with LY294002, compared with the control (Fig. 4E and F). Snail represses E-cadherin expression by directly targeting the promoter of the latter (30). Expression of E-cadherin was also reduced following the addition of LY294002 to the SPC-A1 and the LLC cells, compared with control cells (Fig. 4E and F). These results revealed that miR-126 may inhibit the EMT and metastasis of lung cancer cells via the PI3K/AKT/Snail signaling pathway.

\section{Discussion}

EMT serves a crucial function not only in the development of cancer, but also in cancer progression (31). Cancer cells undergoing EMT are endowed with more aggressive phenotypes than cells that have not undergone EMT, including mesenchymal and stem cell-like features which result in the acquisition of malignant properties, including invasion, metastasis, recurrence and drug resistance. Understanding the molecular mechanisms that regulate EMT is necessary to improve lung cancer treatment. The present study demonstrated that overexpression of miR-126 inhibited TGF- $\beta 1$-induced EMT in lung cancer cells. The present study also demonstrated that regulation of the PI3K/AKT/Snail signaling pathway is involved inmiR-126-mediated EMT and metastasis inhibition in lung cancer cells.

Previous studies have demonstrated that miR-126 inhibits the invasion and migration of lung cancer cells (23). EMT, which is characterized by a loss of cell junctions and the gain of migratory functions, serves a key role in the early process of metastasis of cancer cells. The present study revealed that overexpression of miR-126 inhibited TGF- $\beta 1$-induced EMT. EMT inhibition may be the main mechanism to account for miR-126-mediated inhibition of lung cancer.

Previous studies have reported that miR-126 inhibited the proliferation of lung cancer cells $(20,22)$. However, in the present study there was no significant inhibitory effect on the proliferation of LLC cells in vivo. This may be due to distinct regulatory networks in different types of cancer cell or the complicated microenvironment in vivo. Further studies are needed to address this. Zhang et al (19) reported that miR-126 inhibited breast cancer metastasis by repressing the recruitment of mesenchymal stem cells and inflammatory monocytes. However, in the present study there was no difference in the number or size of metastatic lung cancer tumor nodules between the control and miR-126 overexpression groups. Zhang et al (19) adapted the tail-vein injection assay, which excludes the influence of miR-126 on the cancer cells at the primary tumor site prior to intravasation into the circulation. The present study demonstrated that miR-126 inhibited the mesenchymal-like changes of primary tumor cells, thereby inhibiting the initial step necessary for metastasis.

It has become evident that EMT is one of the numerous cellular processes subject to AKT kinase regulation (28). In addition, TGF- $\beta 1$ induces EMT in human lung cancer cells via the PI3K/AKT and mitogen-activated protein kinase 


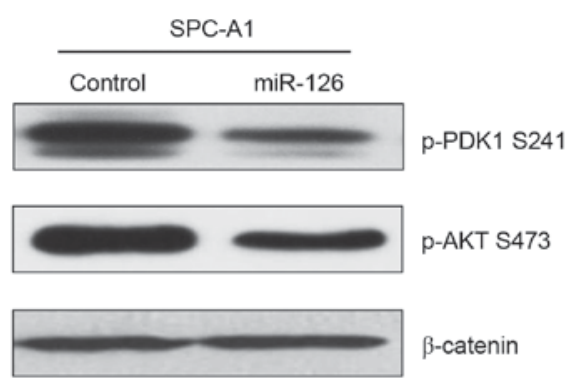

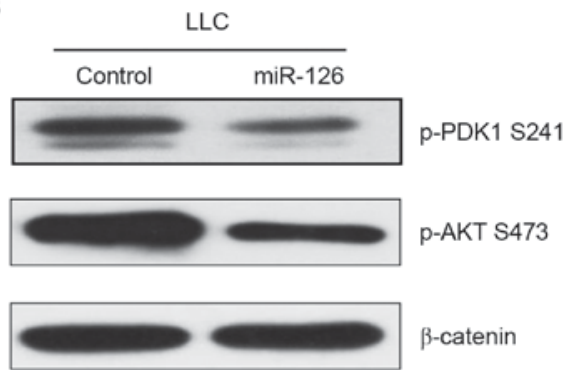

D

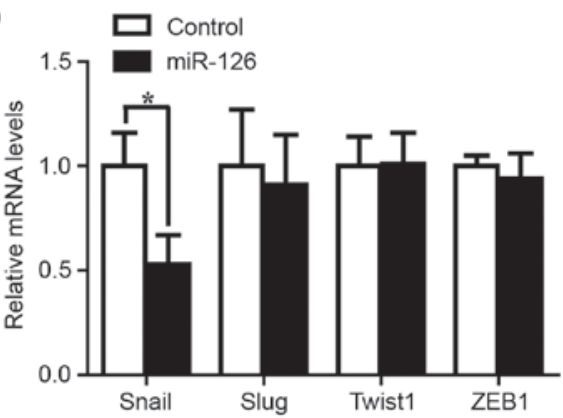

$\mathrm{F}$

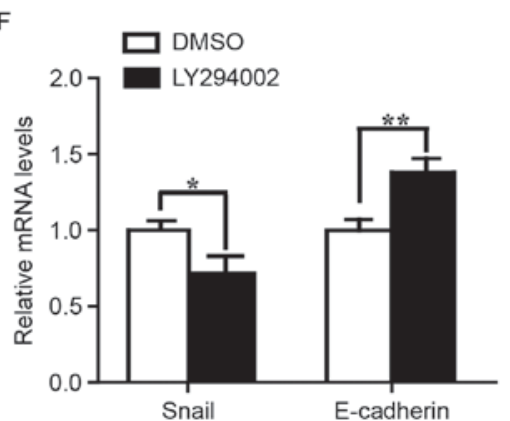

Figure 4. miR-126 directly targets the phosphoinositide 3-kinase/AKT/Snail pathway in lung cancer. Western blot analysis of p-PDK1 and p-AKT expression in (A) SPCA1 and (B) LLC cells transfected with NC and miR-126 mimics. $\beta$-actin was used as an internal control. RT-qPCR analysis of epithelial-mesenchymal-transition-associated gene expression in (C) SPCA1 and (D) LLC cells transfected with NC and miR-126 mimics. RT-qPCR analysis of Snail and E-cadherin expression in (E) SPCA1 and (F) LLC cells following DMSO or LY294002 (50 $\mu \mathrm{M})$ treatment. ${ }^{*} \mathrm{P}<0.05$, ${ }^{* *} \mathrm{P}<0.01 \mathrm{vs}$. control. miR, microRNA; AKT, protein kinase B; p-, phosphorylated; PDK1, pyruvate dehydrogenase kinase 1; NC, negative control; RT-qPCR, reverse transcription-quantitative polymerase chain reaction; E-cadherin, epithelial cadherin.

kinase/extracellular signal-regulated kinase 1/2 signaling pathways (32). EMT is driven by activating AKT and involves upregulation of the mesenchymal cell-specific protein Snail and downregulation of numerous epithelial cell-specific proteins, including E-cadherin (29). SNAIL represses E-cadherin expression by directly targeting the promoter of the latter (30). Previous studies and the present study have demonstrated that miR-126 significantly inhibits the AKT pathway as revealed by reduced p-PDK1 and p-AKT protein. AKT pathway inhibition resulted in reduced Snail expression. Thus, the PI3K/AKT/Snail pathway may be an important means through which miR-126 inhibits the EMT and metastasis of lung cancer cells.

In conclusion, the present study demonstrated that overexpression of miR-126 suppressed the EMT and metastasis of lung cancer cells by targeting the PI3K/AKT/Snail pathway. To the best of our knowledge, this is the first study to demonstrate that miR-126 regulates the EMT of lung cancer cells and also the first to demonstrate that miR-126 inhibits lung cancer metastasis in vivo. However, further studies are required to identify and understand the clinical therapeutic significance of miR-126.

\section{Acknowledgements}

Not applicable.

\section{Funding}

This work was supported by grants from the National Natural Science Foundation of China (grant no. 81541059) and Nanjing Health Bureau (grant no. YKK15065).

\section{Availability of data and materials}

The datasets used and/or analyzed during the current study are available from the corresponding author on reasonable request.

\section{Authors' contributions}

ZJ, YZ, and QX conducted the experiments; WG designed the experiments and wrote the paper; AG helped analyze the data. 


\section{Ethics approval and consent to participate}

In the present study, all methods were subject to approval by the Model Animal Research Center and Ethical Committee of Nanjing University.

\section{Consent for publication}

Not applicable.

\section{Competing interests}

The authors declare that they have no competing interests.

\section{References}

1. Jemal A, Bray F, Center MM, Ferlay J, Ward E and Forman D: Global cancer statistics. CA Cancer J Clin 61: 69-90, 2011.

2. Wood SL, Pernemalm M, Crosbie PA and Whetton AD: Molecular histology of lung cancer: From targets to treatments. Cancer Treat Rev 41: 361-375, 2015.

3. Tse JC and Kalluri R: Mechanisms of metastasis: Epithelial-to-mesenchymal transition and contribution of tumor microenvironment. J Cell Biochem 101: 816-829, 2007.

4. Thiery JP, Acloque $\mathrm{H}$, Huang RY and Nieto MA: Epithelial-mesenchymal transitions in development and disease. Cell 139: 871-890, 2009.

5. Kudo-Saito C, Shirako H, Takeuchi T and Kawakami Y: Cancer metastasis is accelerated through immunosuppression during Snail-induced EMT of cancer cells. Cancer cell 15: 195-206, 2009.

6. Kim AY, Kwak JH, Je NK, Lee YH and Jung YS: Epithelial-mesenchymal transition is associated with acquired resistance to 5-fluorocuracil in HT-29 colon cancer cells. Toxicol Res 31: 151-156, 2015.

7. Singh A and Settleman J: EMT, cancer stem cells and drug resistance: An emerging axis of evil in the war on cancer. Oncogene 29: 4741-4751, 2010.

8. Polyak K and Weinberg RA: Transitions between epithelial and mesenchymal states: Acquisition of malignant and stem cell traits. Nat Rev Cancer 9: 265-273, 2009.

9. Lim LP, Lau NC, Garrett-Engele P, Grimson A, Schelter JM, Castle J, Bartel DP, Linsley PS and Johnson JM: Microarray analysis shows that some microRNAs downregulate large numbers of target mRNAs. Nature 433: 769-773, 2005.

10. Bartel DP: MicroRNAs: Genomics, biogenesis, mechanism, and function. Cell 116: 281-297, 2004.

11. Volinia S, Calin GA, Liu CG, Ambs S, Cimmino A, Petrocca F, Visone R, Iorio M, Roldo C, Ferracin M, et al: A microRNA expression signature of human solid tumors defines cancer gene targets. Proc Natl Acad Sci USA 103: 2257-2261, 2006.

12. Croce CM: Causes and consequences of microRNA dysregulation in cancer. Nat Rev Genet 10: 704-714, 2009.

13. Jiang L, He A, Zhang $\mathrm{Q}$ and Tao C: miR-126 inhibits cell growth, invasion, and migration of osteosarcoma cells by downregulating ADAM-9. Tumour Biol 35: 12645-12654, 2014

14. Zhu N, Zhang D, Xie H, Zhou Z, Chen H, Hu T, Bai Y, Shen Y, Yuan W, Jing Q and Qin Y: Endothelial-specific intron-derived miR-126 is down-regulated in human breast cancer and targets both VEGFA and PIK3R2. Mol Cell Biochem 351: 157-164, 2011

15. Li XM, Wang AM, Zhang J and Yi H: Down-regulation of miR-126 expression in colorectal cancer and its clinical significance. Med Oncol 28: 1054-1057, 2011.
16. Guo C, Sah JF, Beard L, Willson JK, Markowitz SD and Guda K: The noncoding RNA, miR-126, suppresses the growth of neoplastic cells by targeting phosphatidylinositol 3-kinase signaling and is frequently lost in colon cancers. Genes Chromosomes Cancer 47: 939-946, 2008.

17. Zhang J, Du YY, Lin YF, Chen YT, Yang L, Wang HJ and Ma D: The cell growth suppressor, mir-126, targets IRS-1. Biochem Biophys Res Commun 377: 136-140, 2008.

18. Hamada S, Satoh K, Fujibuchi W, Hirota M, Kanno A, Unno J, Masamune A, Kikuta K, Kume K and Shimosegawa T: MiR-126 acts as a tumor suppressor in pancreatic cancer cells via the regulation of ADAM9. Mol Cancer Res 10: 3-10, 2012.

19. Zhang Y, Yang P, Sun T, Li D, Xu X, Rui Y, Li C, Chong M, Ibrahim T, Mercatali L, et al: miR-126 and miR-126* repress recruitment of mesenchymal stem cells and inflammatory monocytes to inhibit breast cancer metastasis. Nat Cell Biol 15: 284-294, 2013.

20. Liu B, Peng XC, Zheng XL, Wang J and Qin YW: MiR-126 restoration down-regulate VEGF and inhibit the growth of lung cancer cell lines in vitro and in vivo. Lung cancer 66: 169-175, 2009.

21. Yang J, Lan H, Huang X, Liu B and Tong Y: MicroRNA-126 inhibits tumor cell growth and its expression level correlates with poor survival in non-small cell lung cancer patients. PLoS One 7: e42978, 2012.

22. Yang X, Chen BB, Zhang MH and Wang XR: MicroRNA-126 inhibits the proliferation of lung cancer cell line A549. Asian Pac J Trop Med 8: 239-242, 2015.

23. Crawford M, Brawner E, Batte K, Yu L, Hunter MG, Otterson GA Nuovo G, Marsh CB and Nana-Sinkam SP: MicroRNA-126 inhibits invasion in non-small cell lung carcinoma cell lines. Biochem Biophys Res Commun 373: 607-612, 2008.

24. Livak KJ and Schmittgen TD: Analysis of relative gene expression data using real-time quantitative PCR and the 2(-Delta Delta C(T)) method. Methods 25: 402-408, 2001.

25. Tiscornia G, Singer O and Verma IM: Design and cloning of an shRNA into a lentiviral silencing vector: Version A. CSH Protoc 2008: pdb.prot5009, 2008.

26. Zhang A, Dong $Z$ and Yang T: Prostaglandin D2 inhibits TGF-beta1-induced epithelial-to-mesenchymal transition in MDCK cells. Am J Physiol Renal Physiol 291: F1332-F1342, 2006.

27. Martelli AM, Evangelisti C, Chiarini F and McCubrey JA: The phosphatidylinositol 3-kinase/Akt/mTOR signaling network as a therapeutic target in acute myelogenous leukemia patients. Oncotarget 1: 89-103, 2010.

28. Lechman ER, Gentner B, Ng SW, Schoof EM, van Galen P, Kennedy JA, Nucera S, Ciceri F, Kaufmann KB, Takayama N, et al: miR-126 regulates distinct self-renewal outcomes in normal and malignant hematopoietic stem cells. Cancer Cell 29: 214-228, 2016.

29. Grille SJ, Bellacosa A, Upson J, Klein-Szanto AJ, van Roy F, Lee-Kwon W, Donowitz M, Tsichlis PN and Larue L: The protein kinase Akt induces epithelial mesenchymal transition and promotes enhanced motility and invasiveness of squamous cell carcinoma lines. Cancer Res 63: 2172-2178, 2003.

30. Nieto MA: The snail superfamily of zinc-finger transcription factors. Nat Rev Mol Cell Biol 3: 155-166, 2002.

31. Larue L and Bellacosa A: Epithelial-mesenchymal transition in development and cancer: Role of phosphatidylinositol 3 kinase/Akt pathways. Oncogene 24: 7443-7454, 2005.

32. Chen XF, Zhang HJ, Wang HB, Zhu J, Zhou WY, Zhang H, Zhao MC, Su JM, Gao W, Zhang L, et al: Transforming growth factor- $\beta 1$ induces epithelial-to-mesenchymal transition in human lung cancer cells via PI3K/Akt and MEK/Erk1/2 signaling pathways. Mol Biol Rep 39: 3549-3556, 2012.

c) (i) $\ominus$ This work is licensed under a Creative Commons Attribution-NonCommercial-NoDerivatives 4.0 International (CC BY-NC-ND 4.0) License. 